\title{
Short communication: Extended-spectrum AmpC-producing Escherichia coli from milk and feces in dairy farms in Brazil
}

\author{
G. S. Santiago, ${ }^{* 1}$ I. S. Coelho, ${ }^{*}$ G. F. Bronzato, ${ }^{*}$ A. B. Moreira,† D. Gonçalves,‡ T. A. Alencar, ${ }^{*}$ H. N. Ferreira, \\ B. G. Castro,† M. M. S. Souza, ${ }^{*}$ and S. M. O. Coelho* \\ *Department of Veterinary Microbiology and Immunology, Federal Rural University of Rio de Janeiro, BR 465, Km 07, Seropédica, RJ, Brazil \\ †Department of Infectious Diseases, Federal University of Mato Grosso, Alexander Ferronato Avenue, 1200, Sinop, MT, Brazil \\ łLaboratory of Biological Science 18.P5.E3, Pharmacy Faculty of University of Porto, Jorge Viterbo Ferreira Street, 228, Porto, Portugal
}

\section{ABSTRACT}

The AmpC enzyme is normally expressed constitutively in Escherichia coli, and its overproduction confers resistance to cefoxitin. A newly reported AmpC, the extended-spectrum AmpC (ESAC), is related to resistance to cefepime, a fourth-generation cephalosporin. This enzyme presents more flexibility in the active site due to insertions, replacements, and deletions on AA sequences. Many isolates producing ESAC were reported in human clinical isolates, but E. coli ESAC producers were reported in animals only in France. The animal E. coli strains can produce this enzyme and possibly disseminate it to human and production environments. In our study, 3 strains of $E$. coli from milk and feces bovine samples, collected in Rio de Janeiro, Brazil, were suspected to produce ESAC. After excluding other mechanisms of resistance, the gene was sequenced to verify ESAC characteristics. These strains presented replacement of AA in omega and R2 loops, suggesting ESAC production. This is the first report to study ESAC E. coli in dairy farms in Brazil.

Key words: AmpC, cefepime, chromosomal gene

\section{Short Communication}

The AmpC enzyme is able to hydrolyze $\beta$-lactams and clavulanic acid, an inhibitor of extended-spectrum $\beta$-lactamase (ESBL), but normally cannot hydrolyze fourth-generation cephalosporins. Cefoxitin is used to differentiate AmpC from other $\beta$-lactamase production in Enterobacteriaceae (Jacoby, 2009; Haenni et al., 2014; Al-Bayssari et al., 2015). However, extended-spectrum AmpC (ESAC) has alarmed health organizations worldwide because it can cause difficulty in human treatments (Rodríguez-Martínez et al., 2012; Pires et

Received August 9, 2017.

Accepted May 8, 2018.

${ }^{1}$ Corresponding author: gabriellissantiago@outlook.com al., 2015). Naturally, AmpC is expressed constitutively at a low level in Escherichia coli and confers resistance to cefoxitin only when it is overproduced, in the case of ESAC production or plasmidic AmpC genes (PeterGetzlaff et al., 2011). The production of ESAC was first detected in human Enterobacter cloacae in 1992. It is characterized by resistance to cefepime, ceftazidime, cefotaxime, and aztreonam, and some hydrolytic activity has been observed on imipenem (Nukaga et al., 1995). Insertions, replacements, or deletions on the 10 or 11 helix, R2 loop, or omega loop can modify the spectrum of enzymes (Barnaud et al., 2001; Mammeri et al., 2004; Nordmann and Mammeri, 2007). These alterations in the AmpC gene lead to conformational changes responsible for more flexibility in the region of the enzyme active site, thereby affecting hydrolytic activity (Nordmann and Mammeri, 2007; Jørgensen et al., 2010). The dissemination of this resistant E. coli may occur between animals and humans by direct transmission or through the food chain (e.g., consumption of unpasteurized cheese; Dierikx et al., 2013; Hammerum et al., 2014; García-Cobos et al., 2015; Wohlwend et al., 2015). Escherichia coli ESAC producers from animal samples were detected in a herd in France (Haenni et al., 2014); there are no data about this strain in production animals in Brazil.

Ten dairy herds from Rio de Janeiro and 20 dairy herds from Mato Grosso, Brazil, were visited between 2009 and 2015 using ethical standards parameters approved by the ethics committee and institutional biosafety committee (protocol no. CEUA-3664040915, Federal Rural University of Rio de Janeiro, Brazil). A total of 185 and $41 \mathrm{E}$. coli strains were obtained from fecal and milk bovine samples, respectively. All isolates were identified by routine biochemical tests and matrixassisted laser desorption/ionization time-of-flight MS assay (Rodrigues et al., 2017; Santiago, 2017). The disk diffusion using amoxicillin, amoxicillin-clavulanic acid, cefoxitin, ceftazidime, cefotaxime, cefepime, aztreonam, and imipenem antibiotics was performed on all $226 \mathrm{E}$. coli strains. The suspected production of ESAC in these 
Table 1. Escherichia coli from milk and feces suspected to produce extended-spectrum AmpC (ESAC) and controls used in this study

\begin{tabular}{llll}
\hline Strain $^{1}$ & Sample/origin & Relevant properties & Source \\
\hline F8 & Feces/Rio de Janeiro & Cefoxitin and cefepim resistance, ESAC suspect & This study \\
F21 & Feces/Rio de Janeiro & Cefoxitin and cefepim resistance, ESAC suspect & This study \\
N18 & Milk/Rio de Janeiro & Cefoxitin and cefepim resistance, ESAC suspect & This study \\
ATCC 25922 & - & Cefoxitin susceptible, control strain & ATCC \\
\hline
\end{tabular}

${ }^{1}$ The nucleotide sequences F8, F21, and N18 have been submitted to the GenBank nucleotide database and have been assigned accession numbers MF361841, MF361842, and MF361843, respectively.

${ }^{2}$ American Type Culture Collection (Manassas, VA).

isolates was based on cefoxitin and cefepime susceptibly testing according to the Clinical and Laboratory Standards Institute (CLSI, 2013, 2014). To exclude ESBL producers, the synergism between amoxicillin-clavulanic acid and third-generation cephalosporins was screened in these strains. The $a m p C$ plasmidic genes were studied by PCR (Pérez-Pérez and Hanson, 2002) and by conjugation in suspected ESAC isolates to observe the ability of the isolates to transmit the gene to other bacteria (Gonçalves, 2008). To detect the mutation in $a m p C$ genes and confirm the production of ESAC, the entire coding region of $a m p C$ was amplified with the primers IntB2 (5'-TTCCTGATGATCGTTCTGCC- $\left.3^{\prime}\right)$ and IntHN (5'-AAAAGCGGAGAAAAGGTCCG-3'), yielding a 1,315-bp amplification product (Mammeri et al., 2006). The PCR reactions were performed with a final volume of $25 \mu \mathrm{L}$ containing $20 \mathrm{~m} M$ Tris- $\mathrm{HCl}$ (pH 8.4), $50 \mathrm{~m} M \mathrm{KCl}, 0.2 \mathrm{~m} M$ deoxynucleoside triphosphates, $1.5 \mathrm{mM} \mathrm{MgCl}_{2}$, and $0.6 \mu M$ each primer; $1.25 \mathrm{U}$ of Taq DNA polymerase (Kapa Biosystems, Fermentas, Brazil) and template DNA $(2 \mu \mathrm{L})$ were added to $23 \mu \mathrm{L}$ of the master mixture. The PCR program consisted of an initial denaturation step at $95^{\circ} \mathrm{C}$ for 5 min followed by 30 cycles of $95^{\circ} \mathrm{C}$ for $1 \mathrm{~min}$, primer annealing at $60^{\circ} \mathrm{C}$ for $1 \mathrm{~min}$, and primer extension at $72^{\circ} \mathrm{C}$ for $1 \mathrm{~min}$. After the last cycle, a final extension step at $72^{\circ} \mathrm{C}$ for 5 min was performed using a T100 Thermal Cycler (BioRad, São Paulo, Brazil). The amplification products were separated by electrophoresis on $2 \%$ agarose gel (Bio-Rad) stained with SYBR Green (Invitrogen, São Paulo, Brazil) and visualized using the photo documentation system L-PIX EX (Loccus Biotecnologia, Cotia, Brazil). The products were purified with Exo-Sap (USB 39 Corp., Cleveland, OH), and the sequencing was performed in ABI 3130xl (Applied Biosystems, São Paulo, Brazil) and edited by DNA Sequence Assembler version 4 (Heracle BioSoft, Arges, Romania) and Mega software version 7 (Caspermeyer, 2016). Escherichia coli ATCC 25922 was used as the control in this study.

Three strains of Escherichia coli from milk (N18) and feces (F8, F21) presented resistance to cefoxitin and cefepime (Table 1). Curiously, these strains were collected from the same farm in Rio de Janeiro in the winter of 2014. Cefoxitin and cefepime resistance is observed in E. coli producing ESAC or coproducing plasmidial AmpC and ESBL (Nordmann and Mammeri, 2007; Jørgensen et al., 2010). To elucidate this, the strains were submitted to conjugation and PCR. The conjugation was evaluated first to detect conjugative AmpC genes, and transconjugants were not found. The PCR excluded the possibility of plasmidial AmpC in these E. coli. After disk diffusion test and interpretative test with third- and fourth-generation cephalosporins and amoxicillin-clavulanic acid disks, no strain demonstrated synergism between $\beta$-lactam and enzyme inhibitors, also excluding the possibility of ESBL producers (CLSI, 2017). Finally, ampC gene sequences were evaluated to confirm the suspicion of ESAC. Escherichia coli ATCC 25922 was used as a negative control to ESAC to compare $a m p C$ gene sequences from strains.

Mammeri et al. (2004, 2008), Sohn et al. (2008), and Bogaerts et al. (2015) reported replacements in the E. coli $A m p C$ gene codifying a different enzyme in human strains. These replacements cause AA substitution in R2, H-9 (close to the R2 loop), and H-10 of AmpC, leading to conformational changes on the enzyme. These loops are considered a catalytic site of AmpC (Mammeri et al., 2004, 2008; Haenni et al., 2014). These modifications cause conformational and flexibility changes in AmpC. Thus, the enzyme can hydrolyze fourth-generation cephalosporins. Only one report describes $E$. coli from animals presenting mainly substitutions in the H-9 helix in the 287 position, causing ESAC production (Haenni et al., 2014). Other substitutions were described by Haenni et al. (2014) but were not related to enzyme modification.

In our study, the relevant AA replacements were in the omega and R2 loops of ESAC suspected strains (Figure 1). All 3 strains showed substitution in the 191 (omega), 209 (omega), and 300 (R2) positions. The omega loop is an important region for enzymes, and 2 substitutions on the omega loop were observed in our study. The first substitution was in the 191 position, and the other was in the 209 position. In the 191 position lysine was substituted for glutamine, and in the 209 position serine was substituted for proline. 


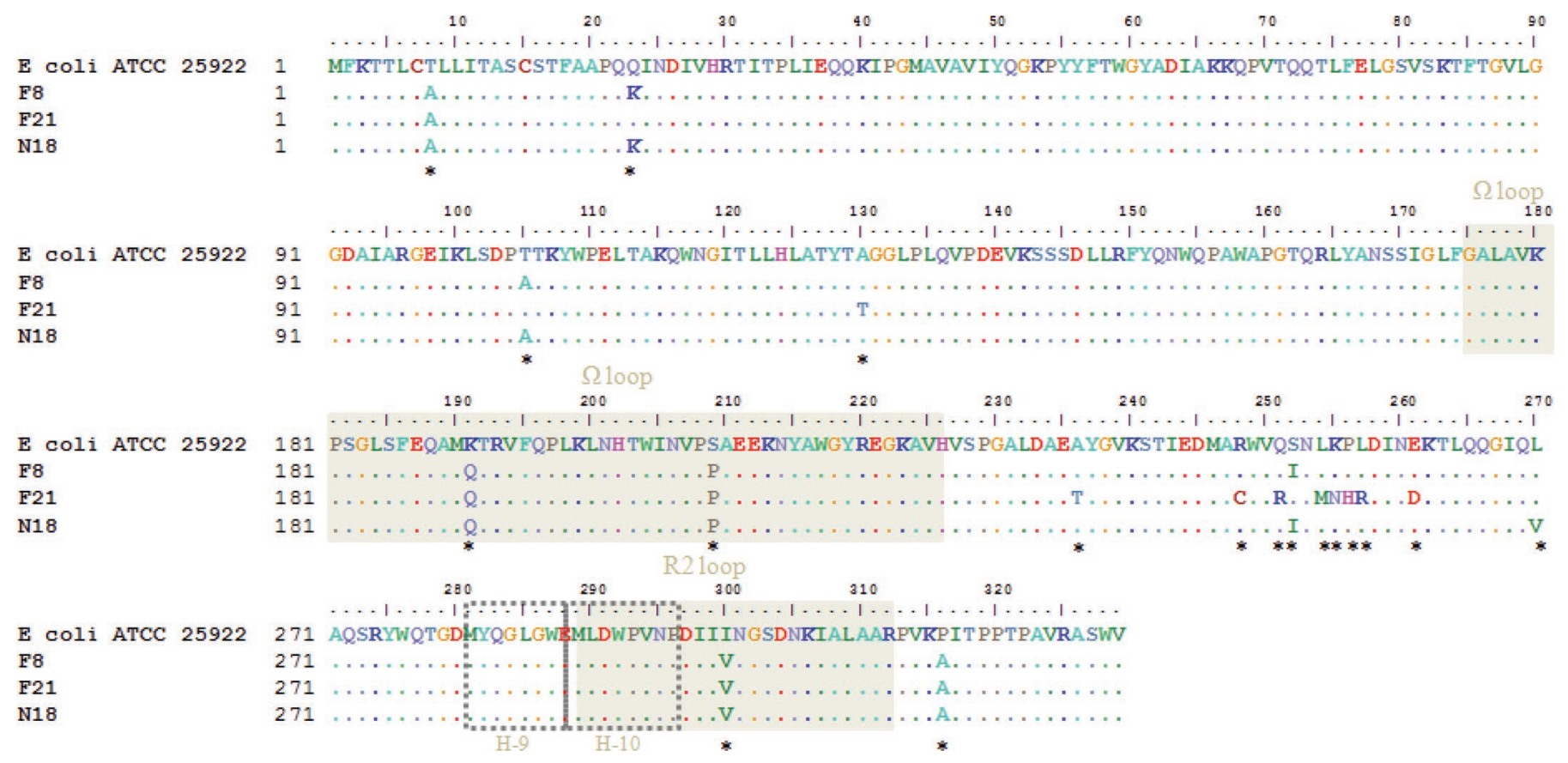

Figure 1. Alignment of the AA sequences of the strains studied. Asterisk $(*)$ indicates that AA replacements were observed in strains suspected to produce extended-spectrum AmpC (ESAC; strains F8, F21, and N18) compared with Escherichia coli ATCC 25922. The shaded boxes represent $\Omega$-loop, H-9 helix, H-10 helix, and R2 loop, respectively. Color version available online.

These AA are not described in the literature, but they have different biochemical characteristics. Lysine is a positive AA, whereas glutamine is negative. This fact is important for secondary, tertiary, and quaternary structures of proteins. In the same way, in position 209 a hydrophilic AA (serine) was replaced by a hydrophobic AA (proline). Proline is located internally in the structure of the enzyme. These variations can cause modifications on the omega loop.

In the R2 region the replacement was observed at the 300 position in all 3 isolates, and the change in AA 298 has already been described in the literature in strains of $E$. coli from human clinical isolates (Mammeri et al., 2006). Other AA variations that have been described are at positions $287(\mathrm{~S} 287 \mathrm{~N}, \mathrm{~S} 287 \mathrm{C}), 292(\mathrm{~A} 292 \mathrm{~V})$, 296 (H296P), 298 (V298L), and 350 (V350F) in isolated human and animal E. coli (Mammeri et al., 2006; Haenni et al., 2014) and 293 (L293P) in human E. coli (Bogaerts et al., 2015). In position 300, the original and replaced AA are hydrophobic (isoleucine to valine). Interactions between AA are important in maintaining enzyme stability, so it is clear that there have been changes suggestive of the change of enzyme spectrum after the observed replacement.

We also found some replacements in the 8, 23, 105, 130, 236, 248, 251, 252, 254, 255, 256, 257, 261, 270, and
316 positions, but these alterations was not observed together in all isolates, as shown in Figure 1. These data were not reported in other animal or human studies. No changes were observed in H-9, as they were not reported by Haenni et al. (2014). Replacements were not observed in $\mathrm{H}-10$, although it has been described in human ESAC E. coli.

Thus, these data of AmpC AA sequences from $E$. coli strains suggest ESAC producers. Although not all replacements have been reported before, because the mutations observed in the coding region of the gene imply AA variations within or near the omega loop and $\mathrm{R} 2$ regions, we propose that cited variations at the 191, 209, and 300 positions can be determinants of ESAC in these isolates. These data demonstrate the possible presence of $E$. coli ESAC in dairy farms in Rio de Janeiro, Brazil. It may be disseminated to other animals and to humans by direct contact in farms or by ingestion of milk that has not been heat treated. In addition, the soil and water in this region may be contaminated with this E. coli.

\section{ACKNOWLEDGMENTS}

This work was financially supported by Foundation for Research Support of the State of Rio de Janeiro 
(FAPERJ, Brazil) and by $\mathrm{PhD}$ sandwich scholarship (Coordination of Improvement of Higher Level Personnel; CAPES) PDSE 99999.008079/2014-05 (Brazil).

\section{REFERENCES}

Al-Bayssari, C., F. Daboussi, M. Hamze, and J. M. Rolain. 2015. Detection of expanded-spectrum $\beta$-lactamases in Gram-negative bacteria in the 21st century. Expert Rev. Anti Infect. Ther. 13:11391158 .

Barnaud, G., R. Labia, L. Raskine, M. J. S. Pors, A. Philippon, and G. Arlet. 2001. Extension of resistance to cefepime and cefpirome associated to a six amino acid deletion in the H-10 helix of the cephalosporinase of an Enterobacter cloacae clinical isolate. FEMS Microbiol. Lett. 195:185-190.

Bogaerts, P., T.-D. Huang, W. Bouchahrouf, C. Bauraing, C. Berhin, F. E. Garch, Y. Glupczynski, and Compath Study Group. 2015. Characterization of ESBL- and AmpC-producing Enterobacteriaceae from diseased companion animals in Europe. Microb. Drug Resist. 21:643-650.

Caspermeyer, J. 2016. MEGA evolutionary software re-engineered to handle today's big data demands. Mol. Biol. Evol. 33:1887.

CLSI (Clinical and Laboratory Standards Institute). 2013. Performance Standards for Antimicrobial Disk and Dilution Susceptibility Tests for Bacteria Isolated from Animals. 2nd Informational Suplemment - 4th ed. CLSI document VET01-A4. CLSI, Wayne, PA.

CLSI (Clinical and Laboratory Standards Institute). 2014. Performance Standards for Antimicrobial Susceptibility Testing; 22nd Informal Supplement. CLSI document M100-S22. CLSI, Wayne, PA.

CLSI (Clinical and Laboratory Standards Institute). 2017. Performance Standards for Antimicrobial Susceptibility Testing. 27th ed. CLSI supplement M100. CLSI, Wayne, PA.

Dierikx, C. M., J. A. van der Goot, H. E. Smith, A. Kant, and D. J. Mevius. 2013. Presence of ESBL/AmpC-producing Escherichia coli in the broiler production pyramid: A descriptive study. PLoS One 8:e79005.

García-Cobos, S., R. Kock, A. Mellmann, J. Frenzel, A. W. Friedrich, and J. W. A. Rossen. 2015. Molecular typing of Enterobacteriaceae from pig holdings in north-western Germany reveals extendedspectrum and AmpC $\beta$-lactamases producing but no carbapenem resistant ones. PLoS One 10:e0134533.

Gonçalves, D. F. M. 2008. $\beta$-Lactamases de espectro alargado em Enterobacteriaceae da flora fecal de idosos. PhD Diss. University of Aveiro, Portugal.

Haenni, M., P. Châtre, and J. Madec. 2014. Emergence of Escherichia coli producing extended-spectrum AmpC $\beta$-lactamases (ESAC) in animals. Front. Microbiol. 5:53.

Hammerum, A. M., J. Larsen, V. D. Andersen, C. H. Lester, T. S. S. Skytte, F. Hansen, S. S. Olsen, H. Mordhorst, R. L. Skov, F. M. Aarestrup, and Y. Agers. 2014. Characterization of extendedspectrum $\beta$-lactamase (ESBL)-producing Escherichia coli obtained from Danish pigs, pig farmers and their families from farms with high or no consumption of third- or fourth-generation cephalosporins. J. Antimicrob. Chemother. 69:2650-2657.

Jacoby, G. A. 2009. AmpC $\beta$-lactamases. Clin. Microbiol. Rev. 22:161182.

Jørgensen, R. L., J. B. Nielsen, A. Friis-Moller, H. Fjeldsoe-Nielsen, and K. Schonning. 2010. Prevalence and molecular characteriza- tion of clinical isolates of Escherichia coli expressing an AmpC phenotype. J. Antimicrob. Chemother. 65:460-464.

Mammeri, H., H. Nazic, T. Naas, L. Poirel, S. Léotard, and P. Nordmann. 2004. AmpC $\beta$-lactamase in an Escherichia coli clinical isolate confers resistance to expanded-spectrum cephalosporins. Antimicrob. Agents Chemother. 48:4050-4053.

Mammeri, H., P. Nordmann, A. Berkani, and F. Eb. 2008. Contribution of extended-spectrum AmpC(ESAC) $\beta$-lactamases to carbapenem resistance in Escherichia coli. FEMS Microbiol. Lett. 282:238-240.

Mammeri, H., L. Poirel, N. Fortineau, and P. Nordmann. 2006. Naturally occurring extended-spectrum cephalosporinases in Escherichia coli. Antimicrob. Agents Chemother. 50:2573-2576.

Nordmann, P., and H. Mammeri. 2007. Extended-spectrum cephalosporinases: Structure, detection and epidemiology. Future Microbiol. 2:297-307.

Nukaga, M., S. Haruta, K. Tanimoto, K. Kogure, K. Taniguchi, M. Tamaki, and T. Sawai. 1995. Molecular evolution of a class C $\beta$-lactamase extending its substrate specificity. J. Biol. Chem. 270:5729-5735.

Pérez-Pérez, F. J., and N. F. Hanson. 2002. Detection of plasmidmediated AmpC- $\beta$-lactamase genes in clinical isolates by using multiplex PCR. J. Clin. Microbiol. 40:2153-2162.

Peter-Getzlaff, S., S. Polsfuss, M. Poledica, M. Hombach, J. Giger, E. C. Böttger, R. Zbinden, and G. V. Bloemberg. 2011. Detection of AmpC $\beta$-lactamase in Escherichia coli: Comparison of three phenotypic confirmation assays and genetic analysis. J. Clin. Microbiol. 49:2924-2932.

Pires, J., M. Taracila, C. R. Bethel, Y. Doi, S. Kasraian, R. Tinguely, P. Sendi, R. A. Bonomo, and A. Endimiani. 2015. In vivo evolution of CMY-2 to CMY-33 $\beta$-lactamase in Escherichia coli ST131: Characterization of an acquired extended-82 spectrum AmpC (ESAC) conferring resistance to cefepime. Antimicrob. Agents Chemother. 59:7483-7488.

Rodrigues, N. M. B., G. F. Bronzato, G. S. Santiago, L. A. B. Botelho, B. M. Moreira, I. S. Coelho, M. M. S. Souza, and S. M. O. Coelho. 2017. The matrix-assisted laser desorption ionization-time of flight mass spectrometry (MALDI-TOF MS) identification versus biochemical tests: A study with enterobacteria from a dairy cattle environment. Braz. J. Microbiol. 48:132-138.

Rodríguez-Martínez, J. M., P. Fernández-Echauri, F. Fernández-Cuenca, P. D. Alba, A. Briales, and A. Pascual. 2012. Genetic characterization of an extended-spectrum AmpC cephalosporinase with hydrolysing activity against fourth-generation cephalosporins in a clinical isolate of Enterobacter aerogenes selected in vivo. J. Antimicrob. Chemother. 67:64-68.

Santiago, G. S. 2017. Compreensão de mecanismos fenotípicos e genotípicos relacionados à produção de $\beta$-lactamases do tipo Ampc em Enterobacteriaceae. MS Thesis. Federal Rural University of Rio de Janeiro, Brazil.

Sohn, S. G., J. J. Lee, E. S. Sohn, L.-W. Kang, and S. H. Lee. 2008. Extension of the hydrolysis spectrum of AmpC $\beta$-lactamase of Escherichia coli due to amino acid insertion in the H-10 helix. J. Antimicrob. Chemother. 61:965-966.

Wohlwend, N., A. Endimiani, T. Francey, and V. Perreten. 2015. Third generation-cephalosporin-resistant Klebsiella pneumoniae isolates from humans and companion animals in Switzerland: Spread of a DHA-producing sequence type 11 clone in a veterinary setting. Antimicrob. Agents Chemother. 59:2949-2955. 